\title{
Effects of Environmental Illuminance on the Readability of E-Books
}

\author{
Tatsuya Koizuka*, Takehito Kojima, Shunta Sano, \\ Nobuhiro Ishio, and Masaru Miyao \\ Nagoya University, Furo-cho, Chikusa-ku, Nagoya, Aichi, 468-8603, Japan \\ koizuka.tatsuya@g.mbox.nagoya-u.ac.jp
}

\begin{abstract}
We carried out experiments to evaluate the readability of e-books using different display systems. We used two types of e-paper (Amazon KindleDX and 300dpiEPD), one type of backlit LCD (new iPad), and ordinary paper text. In the experiments, we conducted subjective evaluations and measured viewing distance. This study found a dependency between readability and illuminance of each device.
\end{abstract}

Keywords: Evaluation of Accessibility, Usability, User Experience, E-books, E-paper, KindleDX, iPad, Readability, Illuminance.

\section{Introduction}

In recent years, e-books have spread to many countries. E-books are available for tablet PCs (e.g. Apple iPad [1]) and E-readers (e.g. Amazon Kindle DX [2]), and are used in various illuminance environments. However, the readability of e-books depends on several factors including the characteristics of the display and environmental illuminance.

In this study subjects evaluated the readability of highly backlit LCDs in low illuminance environments. However, as the illuminance increased so did the reflection of the background light making it more difficult for subjects to read the LCD. In contrast, subjects rated the readability of e-paper as difficult under low illuminance, but as the illuminance increased so did the positive evaluations of readability. Thus, the reading of the backlit LCDs are more difficult to read under high illuminance, while e-paper is easier to read under high illuminance. Because of the increasing popularity of e-paper usage, we were interested in understanding at what exact point of illuminance would be satisfactory for the readability with both forms of media. Our initial conjecture was that this point rests between 1,000 lx and 3,150 lx of illuminance.

\section{Method}

\subsection{Subjects}

The subjects for this study included 130 healthy males and females between the age of 17 and 85 years. Those who needed glasses or contact lenses were permitted to use

\footnotetext{
* Corresponding author. 
them in order to simulate their normal reading situation in normal fashion. We obtained informed consent from all subjects and approval for the study from Ethical Review Board in the Graduate School on Information Science at Nagoya University.

\subsection{Experimental Design}

We carried out the experiments in a darkened room. In order to adjust to constant illumination, we used fluorescent lights and LED lights for reading. We put the reading devices in small compartments on a desk. The subjects read them in comfortable reading posture. In the experiments, we used two types of e-paper (Amazon Kindle DX and 300 dpi EPD), one type of backlit LCD (new Apple iPad) and an ordinary paper text, under five illuminance conditions $(500,750,1,000,2,000,5,000$ lx).
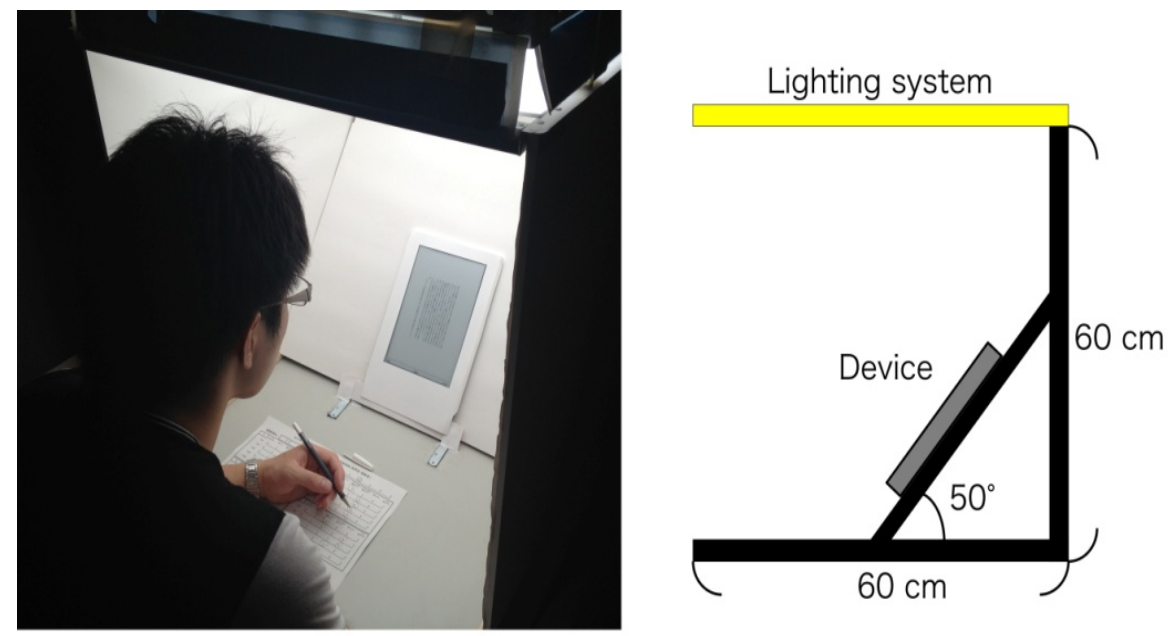

Fig. 1. The reading set up

The picture resolution was 150 dpi (monochrome, 16 gradiation) with Kindle DX, 300 dpi (monochrome, 2 value) with the 300 dpi EPD, 264 dpi (color, 256 gradiation) with the new iPad, and 1200 dpi (monochrome, 2 value) with the paper text. Each textual device was covered with white Kent paper in order to show only the display to the subjects, raised to the same height as the subject on a mounting board, and placed at a 50 degrees angle from the horizontal direction(Figure 1). Table 1 shows the measured illuminance values. 
Table 1. Measured illuminance values $(l x)$

\begin{tabular}{rr}
\hline Illuminance & Measured value \\
\hline $500 l x$ & 565 \\
$750 l x$ & 803 \\
$1,000 l x$ & 1,110 \\
$2,000 l x$ & 2,080 \\
$5,000 l x$ & 5,310 \\
\hline
\end{tabular}

\subsection{Task Design}

The experimental task was to read aloud written text displayed on the devices (Figure 2).There were 34 characters in Japanese per line, and 13 lines in each text passage. The character font was 9-point Shuei-Mincho L. The text style was black/dark on a white/bright background color for screen polarity. The brightness level of the backlight of the LCD was set to the maximum. Table 2 shows the text contrast under the each condition of illuminance.

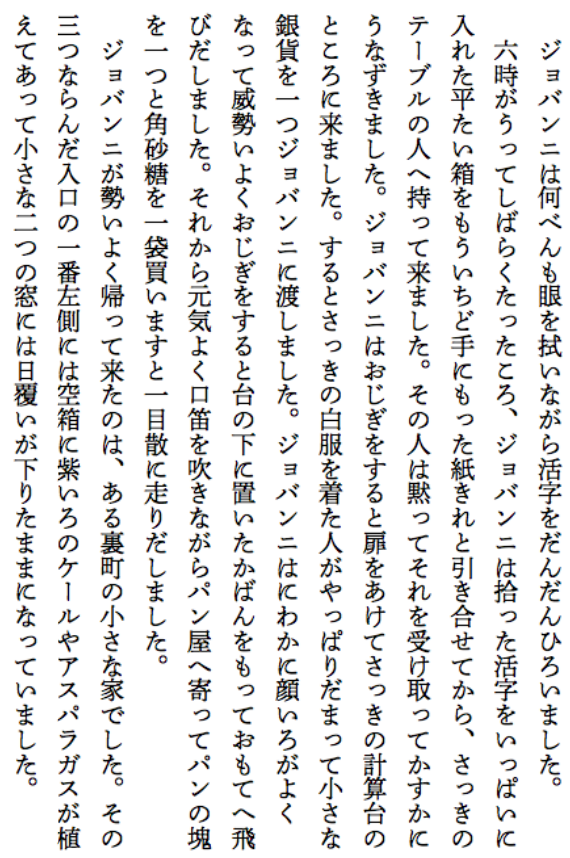

Fig. 2. Example of text for reading task 
In each of the five illuminances $(500-5,000 \mathrm{~lx})$, the participants read aloud a line of text, and we measured their viewing distance with a laser distance meter. After reading, the participants subjectively evaluated the readability of the text. The evaluations by the subjects fell into six groups (Table 3 ).

Table 2. The contrast ratio of each device for given illuminance

\begin{tabular}{rrrrr}
\hline Illuminance & Kindle DX & 300dpiEPD & new iPad & \multicolumn{1}{c}{ paper } \\
\hline $500 l x$ & 8.37 & 7.96 & 151 & 13.3 \\
$750 l x$ & 8.46 & 7.95 & 126 & 13.2 \\
$1,000 l x$ & 8.53 & 8.99 & 99.7 & 13.1 \\
$2,000 l x$ & 8.67 & 7.92 & 53.0 & 13.7 \\
$5,000 l x$ & 8.87 & 8.75 & 26.7 & 13.0 \\
\hline
\end{tabular}

Table 3. Subjective evaluation of readability for tested texts

\begin{tabular}{cc}
\hline Level & Evaluation \\
\hline 0 & Very hard to read \\
1 & Hard \\
2 & A little hard \\
3 & A little easy \\
4 & Easy \\
5 & Very easy \\
\hline
\end{tabular}

\section{$3 \quad$ Results}

\subsection{Subjective Evaluation}

Figure 3 shows the comparison of subjective evaluation for each illuminance. Under $500 \mathrm{~lx}$ and $750 \mathrm{~lx}$, significant differences were observed among e-paper, backlit LCD and paper text. The order from easiest to hardest to read was new iPad, paper text, Kindle DX, and the 300 dpi EPD. Under 1,000 lx, significant differences were observed among e-paper, backlit LCD and paper text. The order from easiest to hardest to read was paper text, new iPad, KindleDX, and the 300dpiEPD. Under 2,000 lx and $5,000 \mathrm{~lx}$, the evaluation of the new iPad was significantly worse than the other devices. The order from easiest to hardest to read was paper text, Kindle DX, 300 dpi EPD, and the new iPad. 


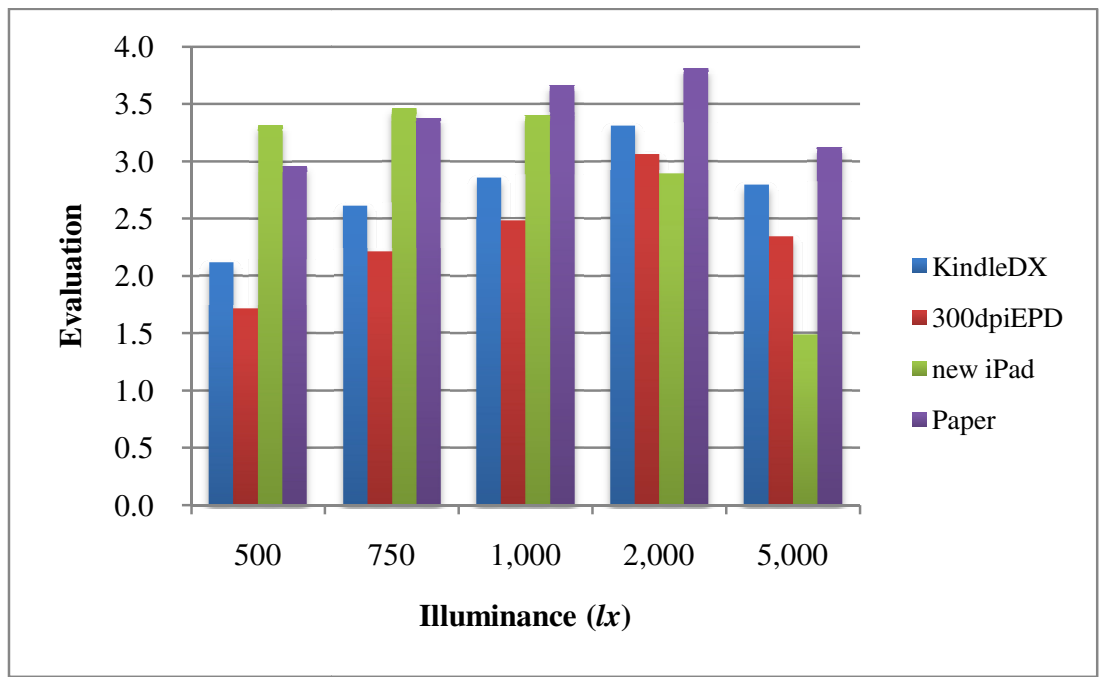

Fig. 3. Subjective evaluation for each illuminance

\subsection{Viewing Distance}

Figure 4 shows the comparison of viewing distance for each illuminace. No significant differences were observed in any conditions. Between $500 \mathrm{~lx}$ and 1,000 $\mathrm{lx}$, the order from furthest to closest was the new iPad, paper text, Kindle DX and 300 dpi EPD. Under 2,000 lx, the order from furthest to closest was the paper text, new iPad, Kindle DX and 300 dpi EPD. Under 5,000 lx, the order from furthest to closest was the paper text, Kindle DX, new iPad, 300 dpi EPD.

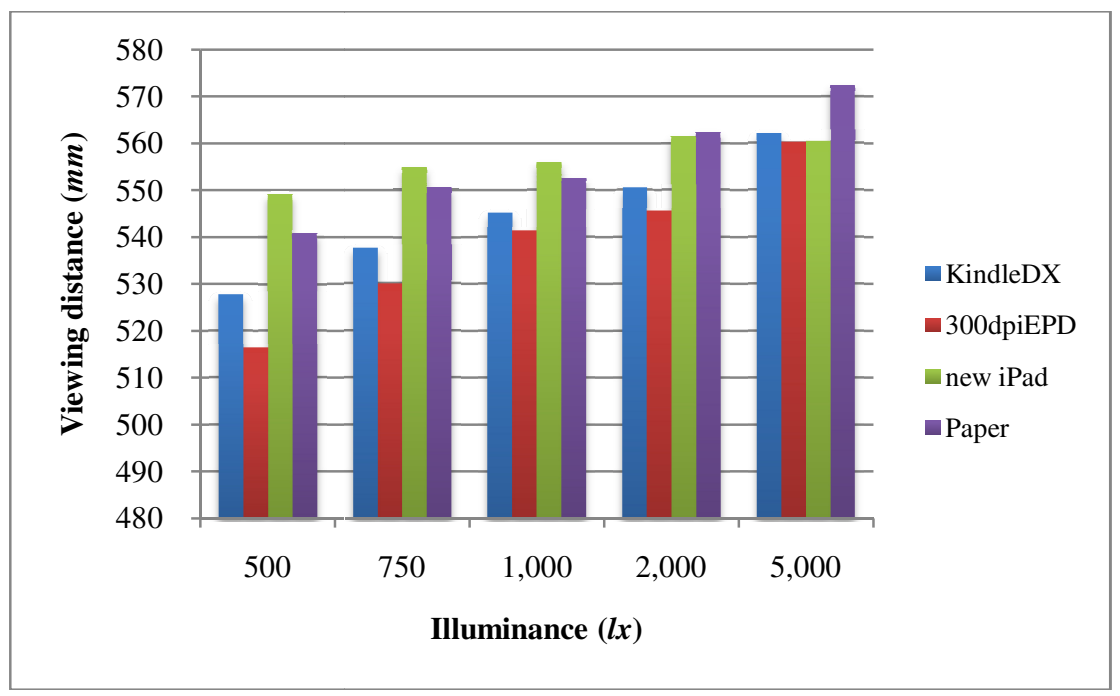

Fig. 4. Viewing distance for each illuminance 


\section{Discussion}

In this study, we evaluated the readability of e-books and ordinary paper text. Under $500 \mathrm{~lx}$ and $750 \mathrm{~lx}$, that is general indoor illuminance, the new iPad was evaluated to be the best. Because the new iPad has a light source and high resolution, readability was evaluated to be better in this condition. When the illuminance became higher than 2,000 lx, the subjects found the Kindle DX was easier to read, and the evaluation of the 300 dpi EPD exceeded that of the new iPad. Under conditions of high illuminance the new iPad cannot prevent glare, and the readability of the new iPad came down.

In terms of viewing distance, the order from furthest to closest was the paper text, the Kindle DX, and 300 dpi EPD, while the distance for the new iPad was found to fluctuate according to illuminace as follows. The viewing distance with the new iPad was the shortest under illuminance of $500 \mathrm{~lx}$, and increased gradually with illuminance up to $2000 \mathrm{~lx}$. Under illuminance of $5000 \mathrm{~lx}$, however, the viewing distance became shorter than that in the case of $2000 \mathrm{~lx}$.

In terms of overall ease of reading, a majority of subjects rated all devices to be easy to read (a little easy, easy, and very easy) under illuminance of 1000 lx and 2000 lx. Under illuminance of 2000 lx, the ease of reading evaluation of the Kindle DX and 300 dpi EPD exceeded that of the new iPad. Under illuminance of 5000 lx, the rating for ease of reading of the new iPad decreased significantly and the new iPad was more difficult to read than other displays and paper.

\section{Conclusions}

When the illuminance was lower than 1,000 1x, the backlit LCD received a better evaluation than that of e-paper. This is because the backlit LCD has its own light source, high resolution and high contrast ratio [3][4]. When the illuminance became higher than 2,000 lx, e-paper received a better evaluation than that of the backlit LCD. This is because glare is prevented with E-paper in high illuminance conditions.

The above results suggest that the crossing point of ease of readability between the Kindle DX and new iPad is approximately illuminance of $1600 \mathrm{~lx}$, and between the 300 dpi EPD and new iPad is approximately illuminance of $1800 \mathrm{~lx}$. The above values were obtained using interpolation without the actual illuminance levels of given values. Accordingly, we may presume that e-paper is superior to backlit LCD in readability when the environmental illuminance is $1800 \mathrm{~lx}$ or more.

Acknowledgments. This research was partially supported by JSPS Kakehnhi (B) Number 24300046 and 23300032.

\section{References}

1. Apple-iPad, http://www . apple.com/ipad/

2. Amazon - Kindle Store,

http: / /www . amazon.com/kindle-store-ebooks-newspapers-

blogs / b/ref=topnav_storetab_kstore? ie=UTF $8 \&$ node=133141011 
3. Kubota, S.: Lightness and contrast requirements for legibility of reflective liquid crystal displays. The Journal of the Institute of Television Engineers of Japan 50, 1091-1095 (1996)

4. Hasegawa, S., Fujikake, K., Omori, M., Matsunuma, S., Miyao, M.: Method to evaluate the legibility of characters on mobile phone LCDs and its application. In: Proceedings of Symposium on Mobile Interactions, pp. 59-64 (2007) 